\title{
Sea Quark and Gluon Polarization in the Nucleon
}

\author{
D. de Florian, G. A. Navarro, and R. Sassot \\ Departamento de Física, Universidad de Buenos Aires, \\ Ciudad Universitaria, Pab.1 (1428) Buenos Aires, Argentina
}

Received on 29 August, 2006

\begin{abstract}
We present results on the quark and gluon polarization in the nucleon obtained in a combined next to leading order analysis to the available inclusive and semi-inclusive polarized deep inelastic scattering data. Using the Lagrange multiplier method, we asses the uncertainty inherent to the extraction of the different spin dependent parton densities in a QCD global fit, and the impact of the increased set of semi-inclusive data now available.
\end{abstract}

Keywords: Semi-Inclusive DIS; Perturbative QCD

\section{INTRODUCTION}

For more than fifteen years, polarized inclusive deep inelastic scattering (pDIS) has been the main source of information on how the individual partons in the nucleon are polarized at very short distances.

Many alternative experiments have been conceived to improve this situation. The most mature among them are those based on polarized semi-inclusive deep inelastic scattering (pSIDIS), i.e. a pDIS experiment where a particular hadron is tagged in the hadronic final state. Choosing different target and final state hadrons, the respective cross sections are sensitive to different combinations of flavored quarks and antiquarks, to be disentangled. These experiments began with the pioneering efforts of SMC, in the late nineties [1], followed by those of HERMES at DESY [2], and lately COMPASS at CERN [3], and are planned to be improved at the Thomas Jefferson National Laboratory (JLAB) in the near future [4].

The phenomenological impact of the pSIDIS data proved to be encouraging although scarce in the initial stage: the reduced number of data and the relatively large estimated errors, at best allowed to check the consistency between pDIS and pSIDIS in a variety of spin-flavor symmetry scenarios [5-7]. With the availability of larger sets of pSIDIS data, much more precise, and for final state hadrons and targets of different flavor composition, the situation now has changed dramatically. pSIDIS data have a non negligible weight in combined global fits at present, comparable to that of inclusive data, and also show clear preferences for the light sea quark polarization. It also helps to constrain the strange sea quark and gluon polarization complementing the information already obtained from pDIS.

In the following we present results obtained in a combined next to leading order analysis to the recently updated set of pDIS and pSIDIS data [8]. Specifically, we focused on the extraction of sea quark and gluon densities, analyzing the constraining power of the data on the individual densities. As result, we found not only a complete agreement between pDIS and pSIDIS data, but a very useful complementarity, leading to rather well constrained densities.

Using the Lagrange multiplier approach [9], we explored the profile of the $\chi^{2}$ function against different degrees of polarization in each parton flavor. In this way we obtained estimates for the uncertainty in the net polarization of each fla- vor, and in the parameters of the pPDFs. We compared results obtained with the two most recent sets of fragmentation functions. The differences are found to be within conservative estimates for the uncertainties. Nevertheless, there is a clear preference for a given set of FF over the other, shown in a difference of several units in the $\chi^{2}$ of the respective global fits. In NLO global fits the overall agreement between theory and the full set of data is sensibly higher than in LO case.

Finally, we analyze the behavior of the cross section for longitudinally polarized proton-proton collisions into neutral pions with a wide range of pPDFs sets coming from a rather conservative uncertainty interval. This observable is found to be crucially sensitive to the polarized gluon density and therefore an invaluable tool. We compute the required precision to be reached in the programed experiments in order to constrain even further this distribution and also future sets of pPDFs. A similar analysis is made for forthcoming pSIDIS data to be obtained at JLAB.

\section{GLOBAL FIT}

In our analysis [8], we followed the same conventions and definitions for the polarized inclusive asymmetries and parton densities adopted in references [6,7], however we used more recent inputs, such as unpolarized parton densities [10] and the respective values for $\alpha_{s}$. In the totally inclusive case, the spin dependent asymmetries are given by:

$$
A_{1}^{N}\left(x, Q^{2}\right)=\frac{g_{1}^{N}\left(x, Q^{2}\right)}{F_{1}^{N}\left(x, Q^{2}\right)}=\frac{g_{1}^{N}\left(x, Q^{2}\right)}{F_{2}^{N}\left(x, Q^{2}\right) / 2 x\left[1+R^{N}\left(x, Q^{2}\right)\right]},
$$

where the inclusive spin-dependent nucleon structure function $g_{1}^{N}\left(x, Q^{2}\right)$ can be written at NLO as a convolution between polarized parton densities for quarks and gluons, $\Delta q_{i}\left(x, Q^{2}\right)$ and $\Delta g\left(x, Q^{2}\right)$, respectively, and coefficient functions $\Delta C_{i}(x)[11]$; $F_{1}^{N}\left(x, Q^{2}\right)$ is the unpolarized nucleon structure function that can be written in terms of $F_{2}^{N}\left(x, Q^{2}\right)$ and $\mathrm{R}$, the ratio of the longitudinal to transverse cross section [12].

Analogously, for the semi-inclusive asymmetries we have:

$$
\left.A_{1}^{N h}\left(x, Q^{2}\right)\right|_{Z} \simeq \frac{\int_{Z} d z g_{1}^{N h}\left(x, z, Q^{2}\right)}{\int_{Z} d z F_{1}^{N h}\left(x, z, Q^{2}\right)},
$$


TABLE I: Inclusive and semi-inclusive data used in the fit.

\begin{tabular}{|c|c|c|c|c|}
\hline Collaboration & Target & Final state & \# points & Refs. \\
\hline EMC & proton & inclusive & 10 & {$[16]$} \\
\hline SMC & proton, deuteron & inclusive & 12,12 & [17] \\
\hline E-143 & proton, deuteron & inclusive & 82,82 & {$[18]$} \\
\hline E-155 & proton, deuteron & inclusive & 24,24 & [19] \\
\hline Hermes & proton,deuteron,helium & inclusive & $9,9,9$ & {$[2]$} \\
\hline E-142 & helium & inclusive & 8 & {$[20]$} \\
\hline E-154 & helium & inclusive & 17 & [21] \\
\hline Hall A & helium & inclusive & 3 & [22] \\
\hline COMPASS & deuteron & inclusive & 12 & [3] \\
\hline SMC & proton, deuteron & $h^{+}, h^{-}$ & 24,24 & {$[1]$} \\
\hline \multirow[t]{2}{*}{ Hermes } & proton, deuteron, helium & $h^{+}, h^{-}, \pi^{+}, \pi^{-}, K^{+}, K^{-}, K^{T}$ & $36,63,18$ & {$[2]$} \\
\hline & Total & & 478 & \\
\hline
\end{tabular}

\begin{tabular}{|c|c|c|c|c|c|c|c|c|c|}
\hline se & & $\chi^{2}$ & $\chi_{D I S}^{2}$ & $\chi_{\text {SIDIS }}^{2}$ & $\delta \bar{u}$ & $\delta \bar{d}$ & $\delta \bar{s}$ & $\delta g$ & $\delta \Sigma$ \\
\hline \multirow{2}{*}{ NLO } & KRE & 430.91 & 206.01 & 224.90 & -0.0487 & -0.0545 & -0.0508 & 0.680 & 0.284 \\
\hline & KKP & 436.17 & 205.66 & 230.51 & 0.0866 & -0.107 & -0.0454 & 0.574 & 0.311 \\
\hline \multirow{2}{*}{ LO } & KRE & 457.54 & 213.48 & 244.06 & -0.0136 & -0.0432 & -0.0415 & 0.121 & 0.252 \\
\hline & KKP & 448.71 & 219.72 & 228.99 & 0.0497 & -0.0608 & -0.0365 & 0.187 & 0.271 \\
\hline
\end{tabular}

where the superscript $h$ denotes the hadron detected in the final state, and the variable $z$ is given by the ratio between the hadron energy and that of the spectators in the target. The region $Z$, over which $z$ is integrated, is determined by kinematical cuts applied when measuring the asymmetries. For the spin dependent structure function $g_{1}^{N}\left(x, Q^{2}\right)$, we use the NLO expression [13]

Fragmentation functions were taken from either [14] or [15], respectively. We also used the flavor symmetry and flavor separation criteria proposed in [14], at the respective initial scales $Q_{i}^{2}$. The data sets analyzed include only points with $Q^{2}>1 \mathrm{GeV}^{2}$, listed in Table I, and totaling 478 data points.

In Table II we summarize the results of the best NLO and LO global fits to all the data listed in Table I. We present fits obtained using alternatively fragmentation functions from reference [14], labeled as KRE, and from reference [15], labeled as KKP.

Since the fit involves 20 parameters, the number of degrees of freedom for these fits is $478-20=458$. Consequently, the $\chi^{2}$ values obtained are excellent for NLO fits and very good for LO. The better agreement between theory and experiment found at NLO, highlights the importance of the corresponding QCD corrections, for the present level of accuracy achieved by the data.

In NLO fits there seems to be better agreement when using KRE fragmentation functions. The difference between the total $\chi^{2}$ values between KRE and KKP NLO fits comes mainly from the contributions related to pSIDIS data, while those associated to inclusive data are almost the same, as one should expect in a fully consistent scenario.

Table II includes also the first moment of each flavor distribution at $Q^{2}=10 \mathrm{GeV}^{2}$, and that for the singlet distribution $\delta \Sigma$, as reference. Most noticeably, while the KRE NLO fit favors the idea of a SU(3) symmetric sea, KKP NLO finds $\bar{u}$ polarized opposite to $\bar{d}$ and to $\bar{s}$. Gluon and strange sea quark polarization are similar in both fits and the total polarization carried by quarks is found to be around $30 \%$.

In Figures 1 and 2 we show the inclusive and semi-inclusive asymmetries computed with the different parameterizations both at LO and NLO accuracy, against the corresponding data sets. The differences between the various sets can hardly be noticed in the comparison to inclusive data in Figure 1, although are more significant when comparing to pSIDIS data, specially in the case of proton targets. This is due to the fact that the main difference between the sets are the light sea quark densities, which are probed by pSIDIS processes of proton targets. The pSIDIS asymmetries for deuterium targets are, of course, less sensitive to these differences since they average $\bar{u}$ and $\bar{d}$ contributions.

\section{UNCERTAINTIES}

Many strategies have been implemented in order to assess the uncertainties in PDFs and their propagation to observables, specially those associated with experimental errors in the data. The Lagrange multiplier method [9] probes the uncertainty in any observable or quantity of interest relating the range of variation of one or more physical observables dependent upon PDFs to the variation in the $\chi^{2}$ used to judge the goodness of the fit to data. In Figure 3 we show the outcome of varying the $\chi^{2}$ of the NLO fits to data against the first moment of the respective polarized parton densities $\delta q$ at $Q^{2}=10$ $\mathrm{GeV}^{2}$, one at a time. This is, to minimize

$$
\Phi\left(\lambda_{q}, a_{j}\right)=\chi^{2}\left(a_{j}\right)+\lambda_{q} \delta q\left(a_{j}\right) \quad q=u, \bar{u}, d, \bar{d}, s, g .
$$




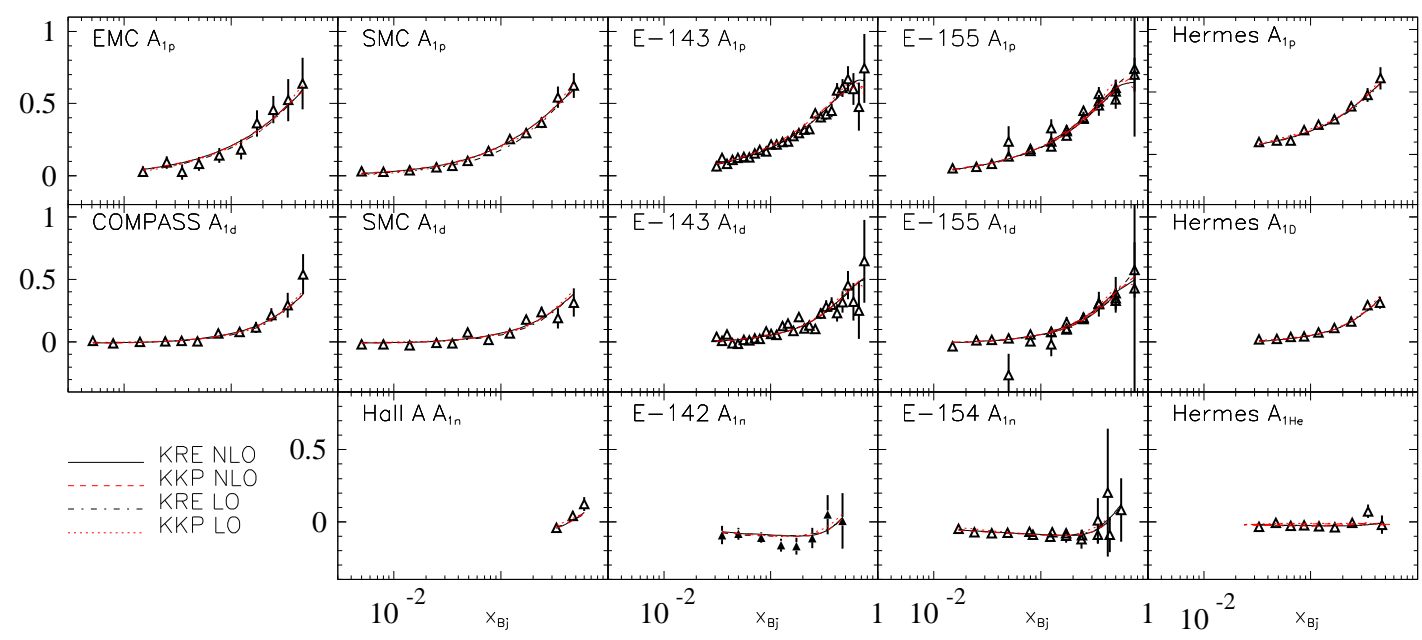

FIG. 1: Inclusive asymmetries computed with LO and NLO pPDFs against the corresponding data.

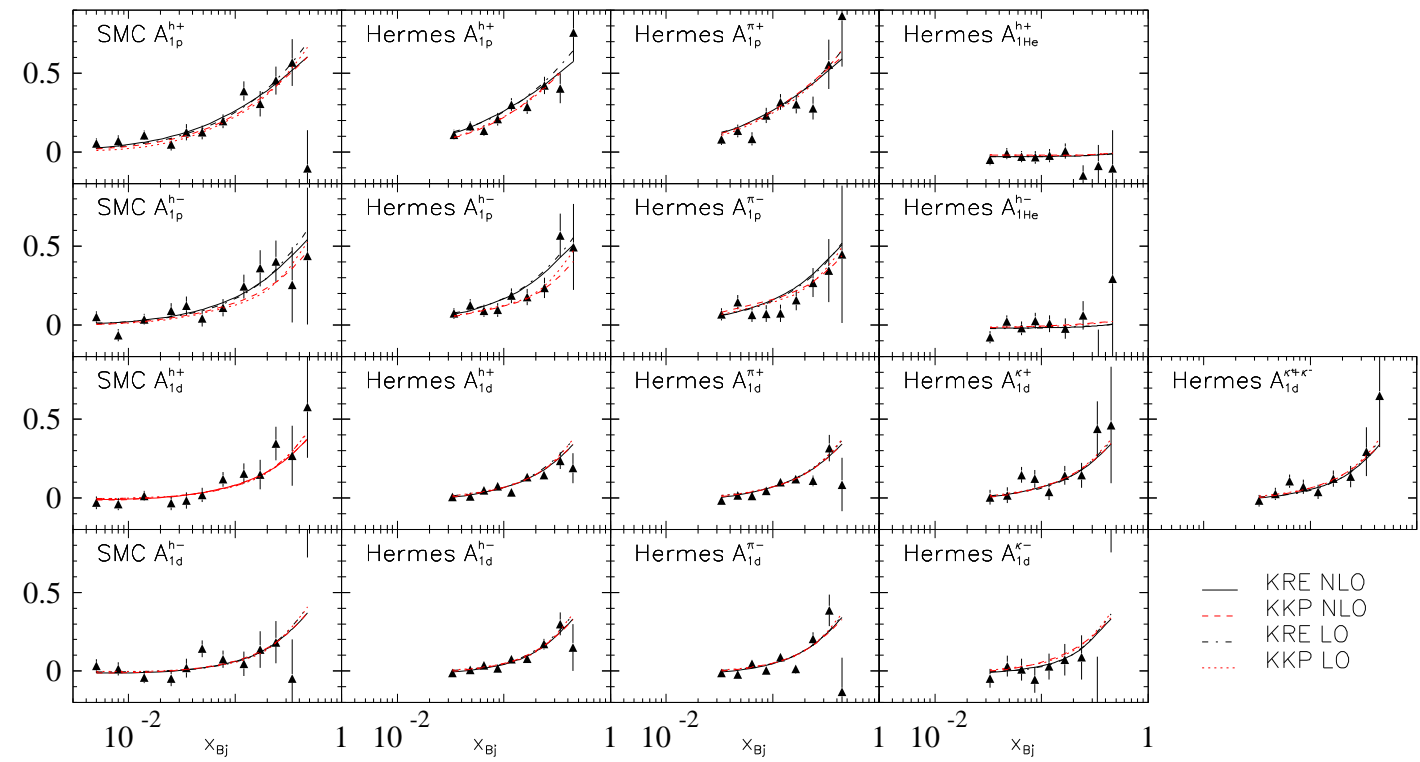

FIG. 2: Semi-inclusive asymmetries computed with LO and NLO pPDFs against the corresponding data.

In order to see the effect of the variation in $\chi^{2}$ on the parton distributions themselves, in Figure 4, we show KRE best fit densities together with the uncertainty bands corresponding to $\Delta \chi^{2}=1$ (darker band) and $\Delta \chi^{2}=2 \%$ (light shaded band). As expected, the relative uncertainties in the total quark densities and those strange quarks are rather small. For gluon densities the $\Delta \chi^{2}=1$ band is also small, but the most conservative $\Delta \chi^{2}=2 \%$ estimate is much more significative. For light sea quarks the $\Delta \chi^{2}=1$ bands are moderate but the $\Delta \chi^{2}=2 \%$ are much more larger. The dot line corresponds to each respective unpolarized parton densities for that values of $Q^{2}$ and $x$, showing that the positivity constraint is fulfilled. 

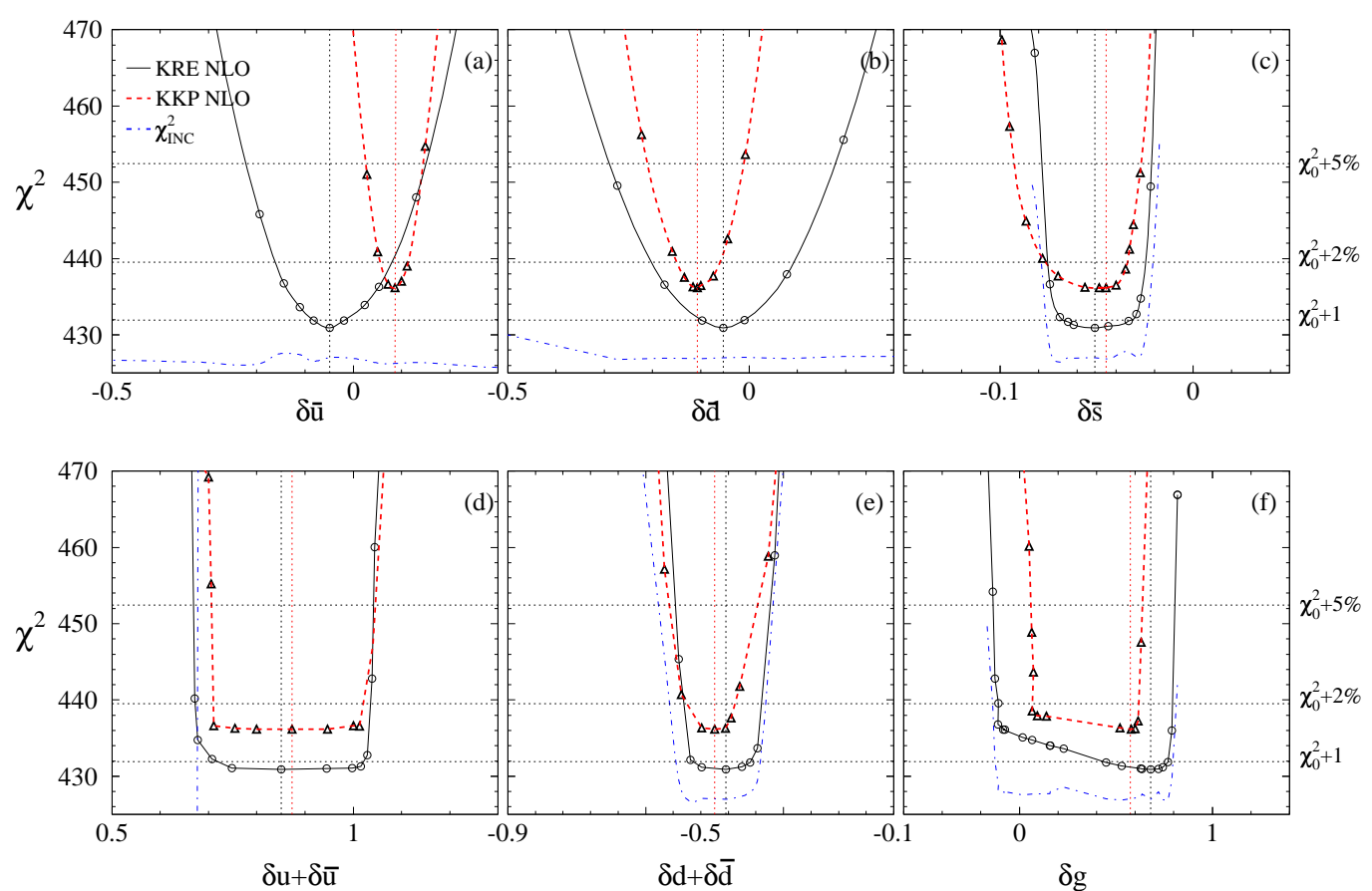

FIG. 3: $\chi^{2}$ profiles for NLO fits obtained using Lagrange multipliers at $Q^{2}=10 \mathrm{GeV}^{2}$.

\section{FUTURE PROSPECTS}

One of the measurements most eagerly awaited by the spin physics community is that of single inclusive large $p_{T}$ pion production in longitudinally polarized proton-proton collisions, which is right now being run at BNL RHIC [23]. The spin dependent asymmetry associated to this kind of process, $A_{L L}^{\pi^{0}}$ is defined, as usual, in terms of the ratio between the polarized and the unpolarized cross sections,

$$
A_{L L}^{\pi^{0}}=\frac{d \Delta \sigma^{p p \rightarrow \pi^{0} X}}{d \sigma^{p p \rightarrow \pi^{0} X}}
$$

which is strongly dependent on the gluon polarization.

The data obtained up to now by the PHENIX Collaboration suggest a very small asymmetry, consistent with pPDFs sets with a moderate gluon polarization. In the following we apply the Lagrange multiplier method in order to explore the range of variation of the estimates for this asymmetry associated to the uncertainty in the present extraction of pPDFs.

In Figure 5 we show the range of variation of $A_{L L}^{\pi^{0}}$ at an intermediate value for $p_{T}=6 \mathrm{GeV}$ obtained with different sets of pPDFs against the variation of the $\chi^{2}$ to pDIS and pSIDIS data for these distributions. The profile of $\chi^{2}$ defines a well defined range of values for $A_{L L}^{\pi^{0}}$ allowed by present pPDFs. The solid line represents the profile of $\chi^{2}$ obtained using KRE FFs, both in the global fit to data and in the computation of the asymmetry. The dashed line represents the same but for KKP FFs. The minima corresponding to both profiles are very close suggesting a cancellation of the associated uncertainty. Notice that both the extraction of the pPDFs, and also the estimate of $A_{L L}^{\pi^{0}}$ with a given set, rely on a set of FFs. The double dependence of the observable on the set of FFs used may had, in principle, even potentiated the disagreement.

In Figure 5 we have included also the profiles obtained using $\delta g$ instead of $A_{L L}^{\pi^{0}}$ in minimization as a dotted line in the case of KRE and a dashed dotted line for KKP. Clearly, the sea quark polarization can conspire in order to yield a larger/smaller asymmetry than the one obtained with maximum/minimum gluon fit at a given $\chi^{2}$, effect which in much more apparent at larger values of $A_{L L}^{\pi^{0}}$. This feature will have to be taken into account in the future for a very precise measurement of the gluon polarization.

Unfortunately, the data collected so far in the first two runs of the PHENIX detector at RHIC have estimated errors much larger than the uncertainties in the values $A_{L L}^{\pi^{0}}$ coming from the fits, however this situation is going to change dramatically in the near future. In Figure 6 we plot $A_{L L}^{\pi^{0}}$ as a function of $p_{T}$ using the best NLO fits coming from KRE and KKP FFs (solid lines and dashes, respectively) and also with KRE variants designed to enhance/reduce $A_{L L}^{\pi^{0}}$ with $\Delta \chi^{2}=2 \%$ (dotted-dashed lines). We have also included the expected uncertainties for the next two runs centered at KRE best fit estimate [24]. Clearly, the future measurement of the asymmetry would certainly be able to constrain even further the gluon and therefore reduce the uncertainties in pPDFs.

Another source of complementary information to further constrain the extraction of pPDFs and also FFs is the exper- 


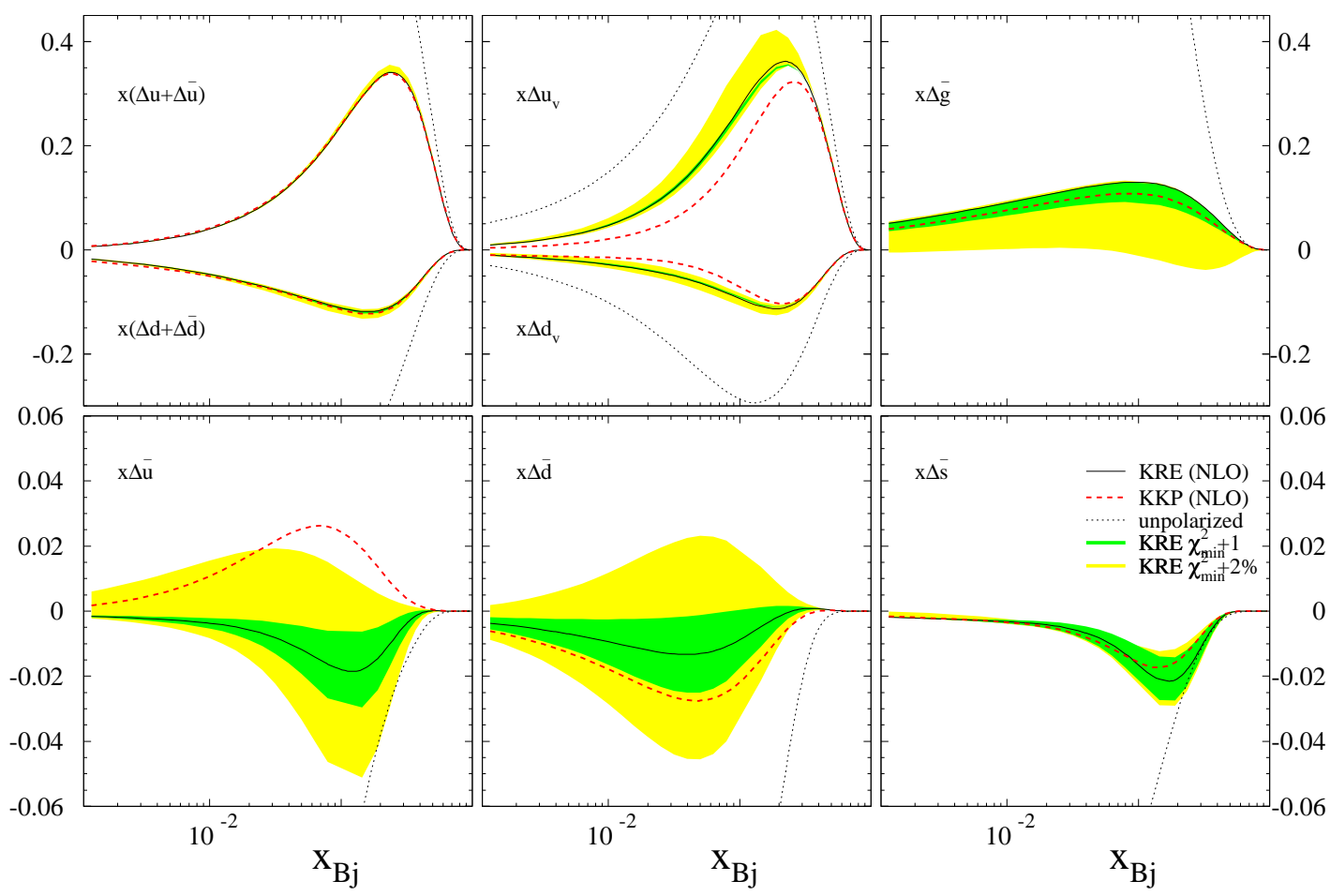

FIG. 4: Parton densities at $Q^{2}=10 \mathrm{GeV}^{2}$, and the uncertainty bands corresponding to $\Delta \chi^{2}=1$ and $\Delta \chi^{2}=2 \%$

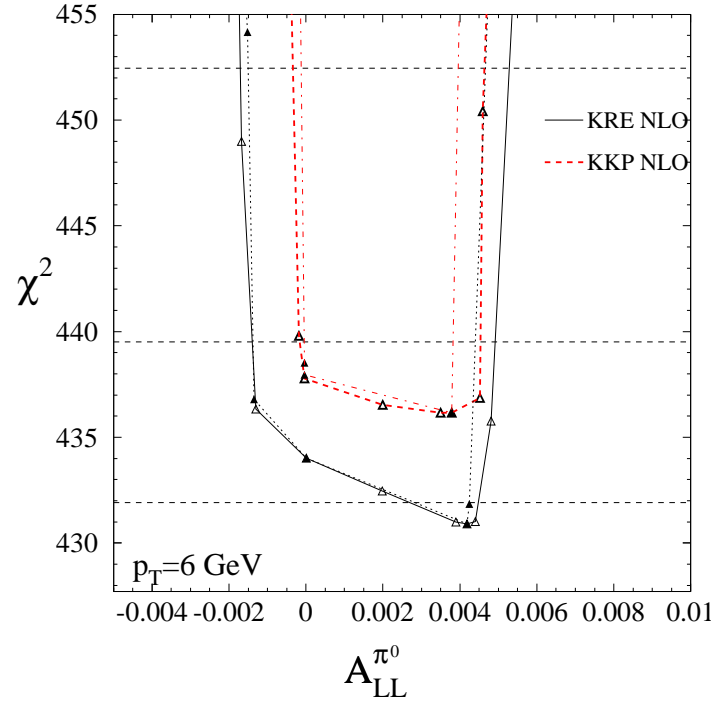

FIG. 5: Profile of the global $\chi^{2}$ to data against $A_{L L}^{\pi^{0}}$ at $p_{T}=6 \mathrm{GeV}$

imental program of the E04-113 experiment at JLAB, which propose to measure pion and kaon pSIDIS asymmetries for proton and deuteron targets [4]. In Figure 7a we show the profile of $\chi^{2}$ of the global fits using KRE (solid line) and

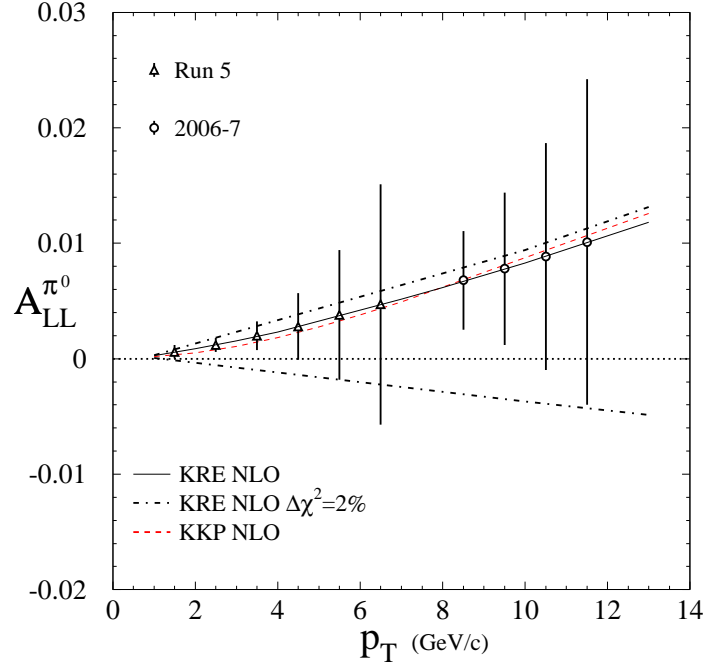

FIG. 6: $A_{L L}^{\pi^{0}}$ vs. $p_{T}$ computed with different pPDF sets and the estimated errors expected by PHENIX at RHIC.

KKP (dashes) FFs against the $\pi^{-}$pSIDIS asymmetry on proton targets at one of the kinematic configurations of E04-113 $\left(x_{B} j=0.203, Q^{2}=2.3 \mathrm{GeV}^{2}<z>=0.5\right)$. The expected 


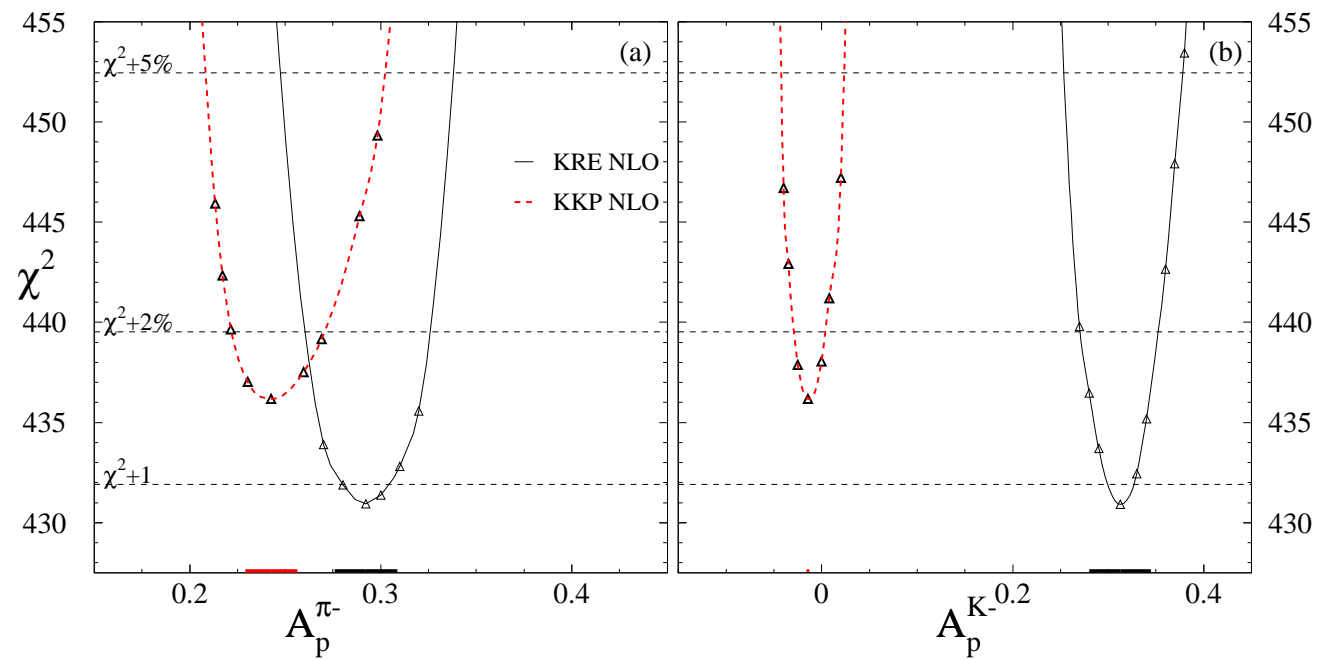

FIG. 7: Profile of the global $\chi^{2}$ to data against $A_{p}^{\pi^{-}}$and $A_{p}^{K^{-}}$proposed to be measured by E04-113 at JLAB.

uncertainty for the measurement of this asymmetry shown in the bottom of the figure, is significantly smaller than those of the previous measurements, and also smaller than the present uncertainty coming from the fit. In Figure $7 b$ we show the same as in the previous figure but but for negative kaons. This asymmetry has not been measured yet and the difference between the predictions coming form KRE (solid line) and KKP (dashes) sets is much larger that the expected uncertainties. The measurement of this last observable together with the combined effect of data for different targets (proton and deuteron) and final state hadrons (positive and negative pions and kaons) will certainly constrain the fit even further, specifically the sea quark densities, and at the same time provide a more stringent test on the quark flavor separation for the FFs used in the analysis.

\section{CONCLUSIONS}

The availability of an important set of new data on polarized processes together with the appropriate theoretical tools required to interpret them, obtained and developed in the last few years, have led the extraction of pPDFs in the proton to mature and to become an important source of information which will still keep growing in the near future.

In this paper, we have assessed the feasibility of obtaining pPDFs, with special emphasis on the sea quark densities, from a combined NLO QCD analysis of pDIS and pSIDIS data. We have estimated the uncertainties associated to the extraction of each parton density, finding a well constrained scenario. pSIDIS data is not only consistent with pDIS, but improves the constraining power of the fit for all the distributions, being crucial for the light sea quarks.

The overall picture found for the quark densities at a 10 $\mathrm{GeV}^{2}$ is one in which, within uncertainties, up quarks are almost $100 \%$ polarized parallel to the proton, down quarks antiparallel in a similar proportion, and sea quarks have a small flavor symmetric negative polarization $[8,25]$

Two programmed experiments, the one based on the PHENIX detector already running at RHIC, and the E04-113 experiment at JLab will be able to reduce dramatically the uncertainty in both the gluon and the light sea quark densities respectively, the latter providing also an even more stringent test on fragmentation functions.

\section{Acknowledgments}

Partially supported by CONICET, Fundación Antorchas, UBACYT and ANPCyT, Argentina. We warmly acknowledges M. B. Gay Ducati, and the Local Comitee of the I Latin American workshop on High Energy Phenomenology for their hospitality during the meeting where this talk was presented.
[1] B. Adeva et al., SMC Collab., Phys. Lett. B 369, 93 (1996), ibid. 420, 180 (1998).

[2] HERMES Collab., A. Airapetian, et al. hep-ex/0407032; K. Ackerstaff et al,, Phys. Lett. B 464, 123 (1999).
[3] A. Bressan, for the COMPASS Collaboration, hep-ex/0501040.

[4] X. Jiang et al. Jefferson Lab experiment E04-113: SemiInclusive Spin Asymmetries on the Nucleon Experiment (hepex/0412010). 
[5] D. de Florian, O. A. Sampayo, and R. Sassot, Phys. Rev. D 57, 5803 (1998).

[6] D. de Florian, R. Sassot, Phys. Rev. D 62, 094025 (2000).

[7] G. A. Navarro and R. Sassot, Eur. Phys. J. C 28, 321 (2003) [arXiv:hep-ph/0302053].

[8] D. de Florian, G. A. Navarro, and R. Sassot, Phys. Rev. D 71 , 094018 (2005).

[9] J. Pumplin, D. R. Stump, and W. K. Tung, Phys. Rev. D 65 , 014011 (2002), D. Stump et al., Eur. Phys. J. C 28, 321 (2003).

[10] A. D. Martin, R. G. Roberts, W. J. Stirling, and R. S. Thorne, Eur. Phys. J. C 28, 455 (2003).

[11] R. Mertig, W. L. van Neerven, Z. Phys. C 70, 637 (1996); W. Vogelsang, Phys. Rev. D 54, 2023, (1996).

[12] B. Lampe and E. Reya, Phys. Rep. 332, 1 (2000); E. Leader et al., Phys, Rep. 261, 1 (1995).

[13] D. de Florian, C.A. Garcia Canal, and R. Sassot, Nucl. Phys. B 470, 195 (1996).

[14] S. Kretzer, Phys. Rev. D 62, 054001 (2000).

[15] B. A. Kniehl, G. Kramer, and B. Potter, Nucl. Phys. B 582, 514 (2000).
[16] EMC Collaboration, J. Ashman et al., Nucl. Phys. B 328, 1 (1989).

[17] SMC Collaboration, B. Adeva et al., Phys. Rev. D 58, 112001 (1998).

[18] E143 Collaboration, K. Abe et al, Phys. Rev. D 58, 112003 (1998).

[19] E155 Collaboration, P. L. Anthony, et al., Phys. Lett. B 463, 339 (1999); G. S. Mitchell, Ph.D. Thesis University of WisconsinMadison, SLAC-Report-540 (1999)

[20] E142 Collaboration, P. L. Anthony, et al., Phys. Rev. D 54, 6620 (1996).

[21] E154 Collaboration, K. Abe et al., Phys. Rev. Lett. 79, 26 (1997); Phys. Lett. B 405, 180 (1997).

[22] HALL A Collaboration X. Zheng, et al., nucl-ex/0405006

[23] S. S. Adler et al. [PHENIX Collaboration], Phys. Rev. Lett. 93, 202002 (2004) [arXiv:hep-ex/0404027].

[24] Y. Fukao [PHENIX Collaboration], hep-ex/0501049.

[25] X. Jiang, G.A. Navarro, and R. Sassot, Eur. Phys J. C 47, 81 (2006). 